\title{
Transverse Spin Relaxation Time
}

National Cancer Institute

\section{Source}

National Cancer Institute. Transverse Spin Relaxation Time. NCI Thesaurus. Code C80364.

The time it takes for the net nuclear spin in the plane that is perpendicular to the magnetic field of a spectrometer to decay to zero. 